\title{
Acceleration of Age-Related Hearing Loss by Early Noise Exposure: Evidence of a Misspent Youth
}

\author{
Sharon G. Kujawa ${ }^{1,2,3,4}$ and M. Charles Liberman ${ }^{1,3,4}$ \\ ${ }^{1}$ Eaton-Peabody Laboratory and ${ }^{2}$ Department of Audiology, Massachusetts Eye and Ear Infirmary, and ${ }^{3}$ Department of Otology and Laryngology, Harvard \\ Medical School, Boston, Massachusetts 02114, and ${ }^{4}$ Division of Health Science and Technology, Harvard University/Massachusetts Institute of Technology, \\ Boston, Massachusetts 02139
}

\begin{abstract}
Age-related and noise-induced hearing losses in humans are multifactorial, with contributions from, and potential interactions among, numerous variables that can shape final outcome. A recent retrospective clinical study suggests an age-noise interaction that exacerbates age-related hearing loss in previously noise-damaged ears (Gates et al., 2000). Here, we address the issue in an animal model by comparing noise-induced and age-related hearing loss (NIHL; AHL) in groups of CBA/CaJ mice exposed identically $(8-16 \mathrm{kHz}$ noise band at 100 $\mathrm{dB}$ sound pressure level for $2 \mathrm{~h}$ ) but at different ages (4-124 weeks) and held with unexposed cohorts for different postexposure times (2-96 weeks). When evaluated 2 weeks after exposure, maximum threshold shifts in young-exposed animals (4-8 weeks) were 40 -50 dB; older-exposed animals ( $\geq 16$ weeks) showed essentially no shift at the same postexposure time. However, when held for long postexposure times, animals with previous exposure demonstrated AHL and histopathology fundamentally unlike unexposed, aging animals or old-exposed animals held for 2 weeks only. Specifically, they showed substantial, ongoing deterioration of cochlear neural responses, without additional change in preneural responses, and corresponding histologic evidence of primary neural degeneration throughout the cochlea. This was true particularly for young-exposed animals; however, delayed neuropathy was observed in all noise-exposed animals held 96 weeks after exposure, even those that showed no NIHL 2 weeks after exposure. Data suggest that pathologic but sublethal changes initiated by early noise exposure render the inner ears significantly more vulnerable to aging.
\end{abstract}

Key words: mouse; noise-induced hearing loss; age-related hearing loss; primary neural degeneration; neuropathy; auditory

\section{Introduction}

Hearing losses that accumulate with chronic exposure to highlevel sound [noise-induced hearing loss (NIHL)] and those we attribute to age [age-related hearing loss (AHL) or presbycusis] are major health problems. They are common, their consequences are permanent, and their impact on human communication and quality of life is significant. NIHL and AHL often coexist in the same ear; however, the conditions under which these forms of hearing loss interact and the mechanisms by which they do so remain poorly understood.

In a recent review of longitudinal hearing loss data from a large cohort of men in the Framingham Heart Study, Gates et al. (2000) observed that, in ears with presumed cochlear damage from previous noise exposure, subsequent hearing loss progression with age was exacerbated at frequencies outside the original NIHL. This observation suggests that ears with noise damage age differently from those without.

This issue of AHL/NIHL interaction has obvious public health

Received Nov. 22, 2005; revised Jan. 6, 2006; accepted Jan. 11, 2006.

This work was supported by National Institutes of Health-National Institute on Deafness and Other Communication Disorders Grants R21 DC04983, R01 DC 00188, and P30 DC 005029. We thank Bruce Tempel for helpful discussions and Melissa Wood for expert technical assistance.

Correspondence should be addressed to Dr. Sharon G. Kujawa, Massachusetts Eye and Ear Infirmary, 243 Charles Street, Boston, MA 02114-3096. E-mail: sharon_kujawa@meei.harvard.edu.

D01:10.1523/JNEUROSCI.4985-05.2006

Copyright $\odot 2006$ Society for Neuroscience $\quad$ 0270-6474/06/262115-09\$15.00/0 significance (Gates et al., 2000; Rosenhall, 2003; Lee et al., 2005) given the high prevalence of noise exposure in and the aging of our society. Concern about long-term effects of noise exposure in young ears is heightened by reports of increasing NIHL prevalence earlier in life (Wallhagen et al., 1997; National Institutes of Health, 2000; Folmer et al., 2002). However, addressing the question in human studies is difficult. Hearing losses in noise-exposed and/or aging ears are highly variable (Gates and Mills, 2005). This variability may arise from underlying differences in actual noise exposures, as well as the influence of other intrinsic and environmental variables that produce hearing loss on their own or alter NIHL vulnerability (Henderson et al., 1993). Such variables do not lend themselves easily to retrospective quantification. Similarly, variability in age of onset, progression, and severity of AHL may be influenced by genetic factors (Gates et al., 1999; DeStefano et al., 2003) and heterogeneity in underlying pathology (Schuknecht and Gacek, 1993), as well as the variable contribution of other insults accumulated over the course of a lifetime (Lutman and Spencer, 1990; Karlsson et al., 1997). This variability has complicated our conduct and interpretation of investigations of NIHL and AHL in humans.

Many of these sources of variability can be eliminated in a laboratory setting using mouse models, in which rigorous genetic and experimental control can be achieved. Indeed, intersubject variability in NIHL within genetically inbred mouse strains is significantly lower than that seen in outbred laboratory animals 
Table 1. Matrix showing the numbers of animals in each of the groups in the present study

\begin{tabular}{cc:c:c:c:c:c:c:c:c}
\hline Unexposed \\
\hline 29 & 11 & 8 & 6 & 11 & 6 & 20 & 5 & 15 & 6 \\
\hline 4 & 6 & 8 & 16 & 32 & 48 & 64 & 80 & 96 & 124 \\
& & & Age (Wks)
\end{tabular}

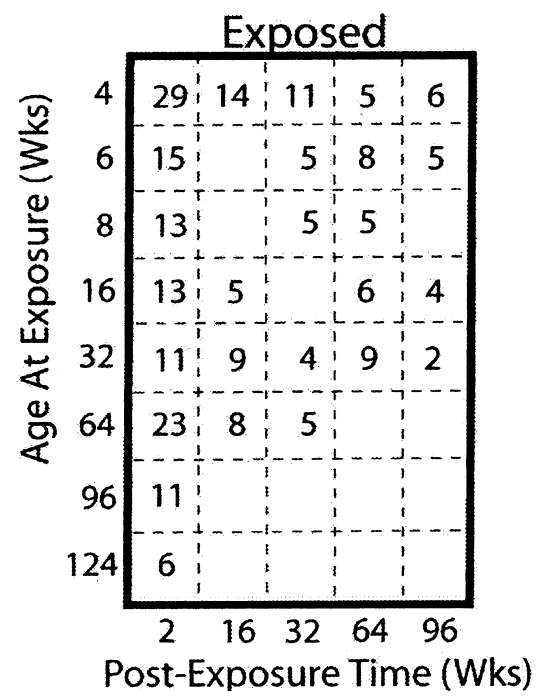

For the "Unexposed" groups, the age indicates the age at final test. For the "Exposed" groups, the row number indicates the age at noise exposure, and the column number indicates the postexposure survival.

(Yoshida et al., 2000; Wang et al., 2002) and humans. Furthermore, important between-strain differences in vulnerability to NIHL and AHL have been identified (Li, 1992; Erway et al., 1993; Johnson et al., 1997; Yoshida et al., 2000; Davis et al., 2003; Candreia et al., 2004). Here, we address the issue of AHL/NIHL interactions directly by comparing NIHL and AHL in groups of CBA/CaJ mice exposed identically but at different ages and held without additional exposure with unexposed cohorts for different postexposure times. Our results suggest that previous noise exposure has significant, deleterious effects on the nature and progression of an age-related hearing loss.

\section{Materials and Methods}

Animals and groups. Mice (CBA/CaJ) of either sex were entered into the protocol at various target ages $(4,6,8,16,32,64,96$, and 124 weeks; $\pm 5 \%$ deviations from targets allowed). Noise exposures were delivered to subsets of animals from each group, and they, along with their unexposed age-matched controls, were held without additional treatment for various postexposure times $(2,16,32,64$, and 96 weeks). Using this strategy, threshold shifts can be compared for animals (1) exposed at the same age but held for different postexposure times, (2) exposed at different ages but held for identical postexposure times, and (3) tested at nominally the same age but exposed and held for different times.

Sound levels in the animal care facility room in which the animals were held were monitored periodically using a data-logging noise dosimeter (NoisePro DLX; Quest Technologies, Oconomowoc, WI). In the periods of monitoring, $24 \mathrm{~h} \mathrm{Leq} \mathrm{[the} \mathrm{equivalent} \mathrm{continuous} \mathrm{sound} \mathrm{level} \mathrm{(Leq)} \mathrm{is}$ a logarithmic average of noise levels in a given area over a stated period of time (e.g., $24 \mathrm{~h}, 1$ year, etc.)] values ranged between $\sim 50$ and $60 \mathrm{~dB}$ sound pressure level (SPL) at the level of the cages. In a final experiment, threshold shifts (NIHL, AHL, and aggregate) were quantified by auditory brainstem responses (ABRs) and distortion product otoacoustic emissions (DPOAEs), and cochlear tissues were recovered from representa- tive animals to characterize the histopathology. The numbers of animals in each group are provided in Table 1. All procedures were approved by the Animal Care Committee of the Massachusetts Eye and Ear Infirmary.

Acoustic overexposures. Exposures $(8-16 \mathrm{kHz}$ octave-band noise, 100 dB SPL, 2 h) were delivered to awake animals held unrestrained within small cells in a subdivided cage (one animal per cell). The noise was generated by a waveform generator (model WG1; Tucker-Davis Technologies, Alachua, FL), filtered $(8-16 \mathrm{kHz}$ bandpass, $>60 \mathrm{~dB} /$ octave slope; Frequency Devices, Haverhill, MA), amplified (D-75 power amplifier; Crown Audio, Elkhart, IN), and delivered (compression driver; JBL, Northridge, CA) through an exponential horn extending into a small, reverberant exposure chamber. The subdivided cage was suspended directly below the horn of the sound-delivery loudspeaker. Noise calibration to target SPL was performed immediately before each exposure session. Sound pressure levels, measured by placing a quarter-inch condenser microphone within each of the four subdivisions of the cage, varied by $<1 \mathrm{~dB}$. Typically, one young ( $4-8$ weeks) animal was included in each exposure session as an additional control for the noise-exposed groups over the many months necessary to accomplish these experiments.

Functional assays. Physiologic tests were conducted in an acoustically and electrically shielded chamber. Animals were anesthetized (ketamine, $100 \mathrm{mg} / \mathrm{kg}$, i.p.; xylazine, $10 \mathrm{mg} / \mathrm{kg}$, i.p.), with booster injections (half of the original dose) given as needed. Temperature was maintained near $37^{\circ} \mathrm{C}$ by heating the air in the experimental chamber. A small V-shaped incision was made in the cartilaginous external canal, widening its opening to facilitate unobstructed viewing of the tympanic membrane and optimum placement of the sound-delivery system.

Stimuli were created and responses were monitored using 16-bit analog-to-digital and digital-to-analog boards (model 6102; National Instruments, Austin, TX) controlled in a LabVIEW environment by a personal computer workstation. Signals used to elicit ABRs and DPOAEs were delivered to the ear using the same custom coupler. The coupler accommodates two transducers (model EC1; Tucker-Davis Technologies) and an EK3103 electret microphone (Knowles Electronics, Itasca, IL) to measure ear-canal sound pressure via a probe tube concentric with the sound-delivery tube. Calibration curves for the probe microphone enabled conversion from voltage to decibel SPL at the probe tip (in decibels relative to $20 \mu \mathrm{Pa}$ ).

ABRs were recorded via subdermal needle electrodes (vertex, ventrolateral to left pinna). Stimuli were $5 \mathrm{~ms}$ tone pips ( $0.5 \mathrm{~ms}$ rise/fall), at frequencies between 5.6 and $45.2 \mathrm{kHz}$ (half-octave steps) delivered at levels below threshold to $80 \mathrm{~dB}$ SPL in $5 \mathrm{~dB}$ steps. Responses were amplified $(10,000 \times)$, filtered $(0.3-3 \mathrm{kHz})$, digitized, and averaged (across 1024 responses at each frequency-level combination; artifact reject, $15 \mu \mathrm{V}$ peak-to-peak). On visual inspection of stacked waveforms, threshold was defined as the lowest stimulus level at which response peaks were repeatably present. Responses absent at the highest level of stimulation $(80 \mathrm{~dB}$ $\mathrm{SPL}$ ) were assigned a threshold value $5 \mathrm{~dB}$ higher. Response values (thresholds, peak-to-peak amplitudes, and N1 latencies) and waveforms were stored to disk for off-line analysis.

DPOAEs were recorded as amplitude versus level functions $\left(\mathrm{L}_{1}, 20-75\right.$ or $80 \mathrm{~dB}$ SPL in $5 \mathrm{~dB}$ steps; $\left.\mathrm{L}_{2}, \mathrm{~L}_{1}-10\right)$ at $\mathrm{f}_{2}$ frequencies $\left(\mathrm{f}_{2} / \mathrm{f}_{1}=1.2\right)$ between 5.6 and $45.2 \mathrm{kHz}$ (half-octave spacing). Ear-canal sound pressure was amplified and digitally sampled at $4 \mu$ s intervals. DPOAE amplitude at $2 f_{1}-f_{2}$ and surrounding noise floor $\pm 50 \mathrm{~Hz}$ of the DPOAE were extracted from the averaged waveforms of ear-canal sound pressure. DPOAE and noise floor values and averaged waveforms were stored to disk. Responses were analyzed as iso-response functions relative to L2 levels required to generate DPOAEs of $-5 \mathrm{~dB}$ SPL (Kujawa and Liberman, 1999). Stimulus levels were kept below $80 \mathrm{~dB}$ SPL to avoid systemgenerated distortion. When responses were absent at the maximum levels presented, a threshold value $5 \mathrm{~dB}$ higher was again assigned. The $\mathrm{ABR}$ stimulus-level maximum was set to $80 \mathrm{~dB}$ SPL for consistency.

Histologic preparation and analyses. After final physiological testing, selected animals were deeply anesthetized, and cochlear tissues were retrieved for histologic processing and evaluation. Ears were prepared by a thick-sectioning technique allowing thorough light microscopic evaluation of all structures of the murine cochlea (Hequembourg and Liberman, 2001). In brief, animals were perfused intracardially with $2.5 \%$ 
glutaraldehyde and $1.5 \%$ paraformaldehyde in phosphate buffer. Both cochleas were extracted, and the round and oval windows were opened to allow intra-labyrinthine perfusion of the same fixative. After overnight postfixation in the same fixative at $4^{\circ} \mathrm{C}$, the cochleas were osmicated $(1 \%$ $\mathrm{OsO}_{4}$ in $\mathrm{dH}_{2} \mathrm{O}$ ) for $1 \mathrm{~h}$ and then decalcified $(0.1 \mathrm{~m}$ EDTA with $0.4 \%$ glutaraldehyde) for 2-3 d. Decalcified cochleas were dehydrated in ethanol and propylene oxide, embedded in Araldite resins, and sectioned at $40 \mu \mathrm{m}$ on a Historange with a carbide steel knife. Sections were mounted in Permount on microscope slides and coverslipped.

Cochlear structures were assessed at the light microscopic level for signs of histopathology. We used a semiquantitative rating scale for assessment of fractional cellular survival of the sensory cells and their afferent innervation (spiral ganglion cells), as well as for cellular elements within three critical accessory structures of the cochlear duct: the stria vascularis, spiral ligament, and spiral limbus. The examiner was blind to age-exposure status.

\section{Results}

The CBA/CaJ mouse was chosen for this study because, in contrast to many other inbred strains (Zheng et al., 1999), it maintains good threshold sensitivity well into old age (Henry and Chole, 1980; Hunter and Willott, 1987; Jimenez et al., 1999). Two metrics of auditory function were used to measure threshold sensitivity: DPOAEs and ABRs. DPOAEs arise from normal cochlear nonlinearities generated by transduction in outer hair cells and are not affected by damage to inner hair cells or cochlear neurons (Liberman et al., 1997). ABRs represent the summed activity of auditory neurons and thus require functional integrity of all preneural elements (including both outer and inner hair cells), as well as their afferent innervation. Comparison of threshold shifts seen via the two measures thus provides important clues as to the site(s) of dysfunction. Young adult animals tested here had baseline thresholds similar to those reported previously (Henry, 2004).

\section{NIHL vulnerability varies with age at exposure}

Effects of noise exposure include reversible and irreversible components. After exposures that are intense enough to produce permanent effects, thresholds recover exponentially with increasing postexposure time and reach steady state within $\sim 2$ weeks (Miller et al., 1963). Thus, to evaluate vulnerability to permanent NIHL, threshold shifts were initially measured at 2 weeks after exposure.

Age at exposure was varied systematically while holding all other exposure parameters (sound pressure, duration, bandwidth, etc.) constant, to examine its influence on NIHL. Figure 1 shows mean threshold shifts for animals at two extremes of our exposure-age range (4 vs 96 weeks). Young-exposed animals show a maximum threshold shift of $\sim 40 \mathrm{~dB}$ at the frequency ( 16 $\mathrm{kHz}$ ) corresponding to the upper edge of the exposure band $(8-16 \mathrm{kHz})$. In contrast, old-exposed ears show no threshold elevation. Similar results were obtained with ABR and DPOAE measures, consistent with the notion that the functionally important changes in these ears involve the outer hair cells, which are among the most vulnerable structures in the inner ear (Hamernik et al., 1989; Saunders et al., 1991; Dallos, 1992).

A more detailed look at the relationship between age and noise vulnerability is offered in Figure 2. Here, maximum threshold shift at 2 weeks (i.e., shift at $16 \mathrm{kHz}$ ) is plotted versus age at exposure for all groups in the present study (Fig. $2 A, B$ ). The data show a dramatic shift in vulnerability between 8 and 16 weeks: there is little difference among the young-exposed groups (4, 6, and 8 weeks) and little difference among the older-exposed groups $(16,32,64$, and 124 weeks) at this 2 week postexposure
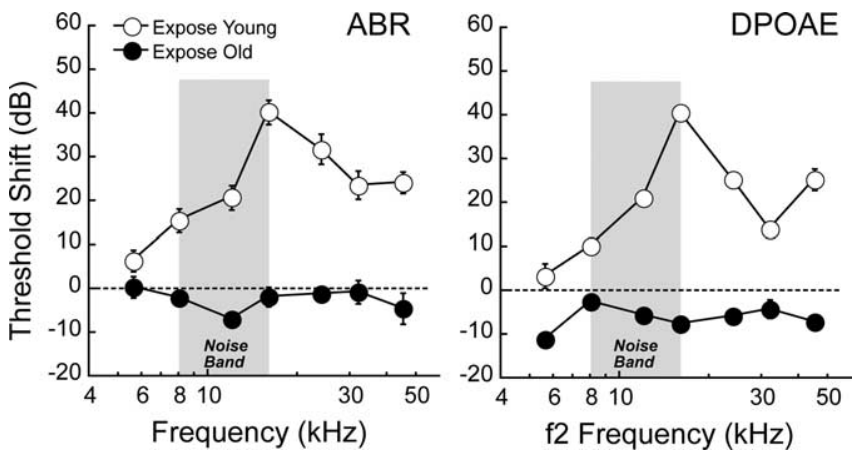

Figure 1. Young (4-8 weeks) mice are more vulnerable to noise damage than old (96 weeks) mice. Each age group was exposed to high-level noise, and threshold shifts were measured by ABR and DPOAE 2 weeks later. Threshold shifts (calculated relative to age-matched, unexposed cohorts) are greater in young-exposed ears by both measures. Data are expressed as means \pm SE. For the numbers of animals in each group, see Table 1 . The gray bar denotes the pass band of the noise-exposure stimulus.
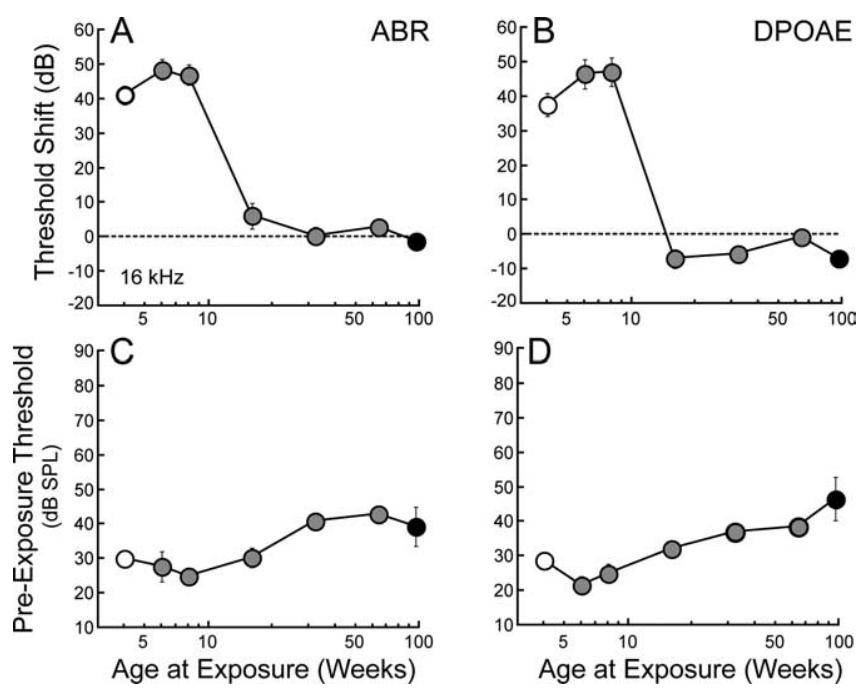

Figure 2. Vulnerability to noise decreases dramatically between 8 and 16 weeks of age. $\boldsymbol{A}, \boldsymbol{B}$, Maximum threshold shifts (i.e., shifts at $16 \mathrm{kHz}$ ) seen at 2 weeks after exposure by $A B R(\boldsymbol{A})$ and DPOAE $(\boldsymbol{B})$ for all ages at exposure. $\boldsymbol{C}, \boldsymbol{D}$, In unexposed control ears, thresholds at $16 \mathrm{kHz}$ do not show large change between 8 and 16 weeks. Data are means \pm SE and are plotted as a function of age on a logarithmic scale. For the numbers of animals in each group, see Table 1.

time. DPOAE and ABR data are virtually identical, and there were no statistically significant gender differences in NIHL.

It is important to consider the age-related progression in preexposure thresholds over the same timespan, shown in Figure 2, $C$ and $D$. The precipitous drop in maximum noise-induced threshold shift between 8 and 16 weeks has no obvious counterpart in a change in baseline sensitivity: our data from unexposed groups show that sensitivity at $16 \mathrm{kHz}$ changes $<5$ and $<10 \mathrm{~dB}$ across the entire frequency range of test over the same period of time.

\section{AHL is exacerbated by previous noise exposure}

To evaluate interactions between NIHL and subsequent AHL, we tracked thresholds in noise-exposed versus unexposed ears with increasing postexposure survival, out to the lifespan of the mouse $(\sim 2.5$ years $)$.

First, consider animals exposed to noise at 4 weeks. When measured 2 weeks later, threshold shifts by both $\mathrm{ABR}$ and DPOAE peaked at $40 \mathrm{~dB}$ at $16 \mathrm{kHz}$ (open circles in Fig. $3 \mathrm{~A}, \mathrm{~B}$ are 

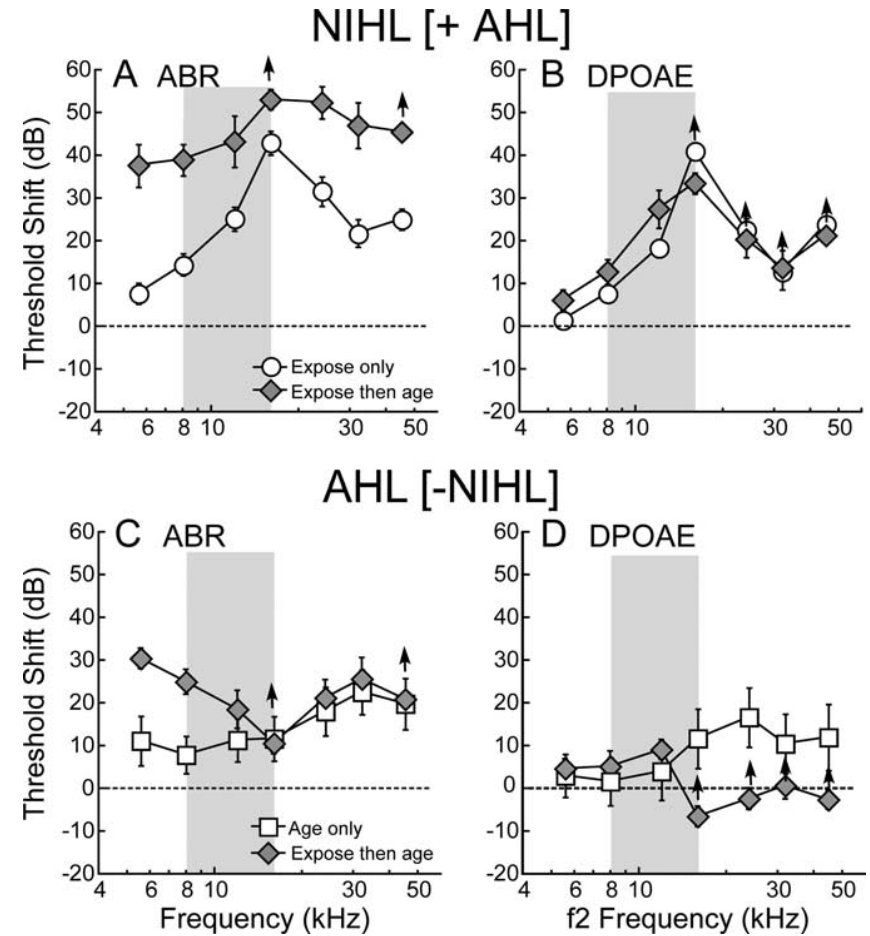

Figure 3. Early NIHL exacerbates AHL when measured by $\operatorname{ABR}(\boldsymbol{A}, \boldsymbol{C})$ but not by $\operatorname{DPOAE}(\boldsymbol{B}, \boldsymbol{D})$. $A, B$, NIHL in animals exposed at 6 weeks (white circles; replotted from Fig. 1 ) is defined as thresholds at 2 weeks after exposure relative to unexposed 6 week controls; 96 weeks later, aggregate NIHL/AHL in animals exposed at 6 weeks is also calculated relative to unexposed 6 week controls. $\boldsymbol{C}, \boldsymbol{D}, \mathrm{AHL}$ in unexposed animals (white squares) is simply the difference between thresholds at 102 versus 6 weeks; AHL for the noise-exposed group (gray diamonds) removes the initial NIHL component, i.e., it is the difference between thresholds at 96 versus 2 weeks after exposure (the difference between the curves in $\boldsymbol{A}$ and $\boldsymbol{B}$ ). Data are means $\pm \mathrm{SE}$. For the numbers of animals in each group, see Table 1 . The arrows above the points indicate that at least $50 \%$ of animals from this group at this frequency lacked responses at the highest SPLs presented; thus, the threshold shift may be underestimated. For additional explanation, see $\mathrm{Ma}-$ terials and Methods.

replotted from Fig. $1 A, B)$. When reexamined 96 weeks later, ABR threshold shifts had grown dramatically across the entire range of test frequencies, whereas DPOAE thresholds changed only slightly (Fig. $3 A, B$ ). This striking discrepancy between ABR and DPOAE shifts suggests that progressive age-related changes in the noise-damaged ear involve the inner hair cell and/or the auditory nerve to a greater degree than the outer hair cells or other structures contributing to the cochlear amplifier. The difference between threshold curves at 2 versus 96 weeks after exposure is a measure of the AHL in these young-exposed ears. As seen in Figure $3 C$, this AHL (filled squares) is significantly larger than that seen in unexposed ears (open squares) when evaluated by ABR. Thus, early noise exposure increases AHL, especially at frequencies below the region of maximum damage (e.g., 5.6 and 8.0 $\mathrm{kHz}$ ), in which initial postexposure threshold shift was minimal. Note that the exacerbation of AHL at high frequencies may be even greater than suggested, given that both ABR and DPOAE measures of threshold shift "saturate" as the sound pressures required to elicit a response (in the noise-exposed groups) reach the maximum sound pressures tested (Fig. 3, upward arrows) (for additional explanation, see Materials and Methods). With respect to possible gender effects of AHL/NIHL interactions, the number of males in the unexposed, old group was too small $(n=$ 2) to calculate meaningful threshold shifts.

To evaluate how the interaction between AHL and NIHL var-
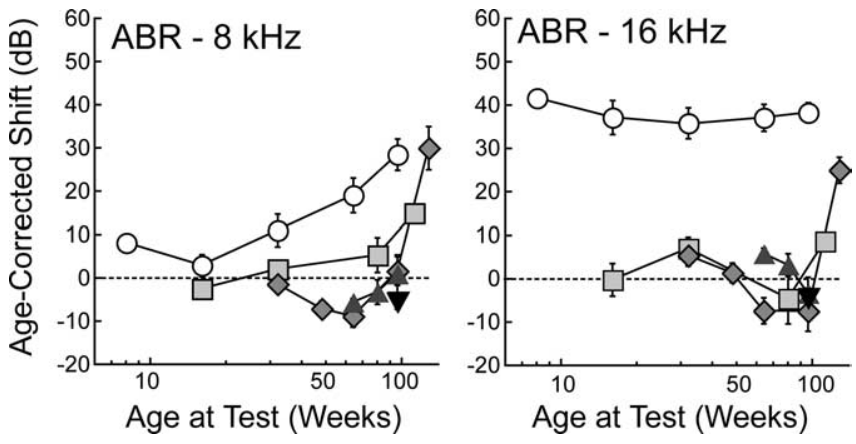

Figure 4. Progressive threshold shifts as a function of age in animals initially exposed to noise at different ages: 6 weeks (white circles), 16 weeks (gray squares), 32 weeks (gray diamonds), 64 weeks (gray triangles), or 96 weeks (black triangles). Shifts are shown at $16 \mathrm{kHz}$ (right), the frequency of maximum initial shift, and $8 \mathrm{kHz}$ (left), a frequency showing minimal initial shift. Age-corrected shifts are defined as the difference between the measured threshold and the thresholds of unexposed animals of similar age. Data are plotted as a function of age at test; thus, an animal exposed at 16 weeks and held for 32 weeks will be plotted at 48 weeks of age. The numbers of animals in each group are given in Table 1.

ies with age-of-exposure, we extract the ABR-based threshold shifts at 8 and $16 \mathrm{kHz}$ from each exposed group at each postexposure age and "correct" for age by subtracting the corresponding threshold shift seen in age-matched, unexposed controls (Fig. 4). By this procedure, the "age-corrected shift" is a measure of the original noise-induced shift plus any additional hearing loss seen in the ABR response, above and beyond that expected attributable to aging alone. Consider first the data at $8 \mathrm{kHz}$. For the group exposed at 4 weeks (open circles), the age-corrected shift grows steadily with postexposure time to a maximum of $\sim 25 \mathrm{~dB}$ at 96 weeks (the same value shown in Fig. $3 \mathrm{C}$ at $8 \mathrm{kHz}$ ), suggesting a strong interaction between NIHL and subsequent AHL. Data from the groups exposed at 16 and 32 weeks (Fig. $4 A$, squares and diamonds, respectively) also show interactions between NIHL and AHL at the longest postexposure holding times: the thresholds deteriorate after noise exposure more dramatically than in age-matched, unexposed counterparts. Thus, the long-term sequelae of noise exposure are visible regardless of whether the exposure occurred before or after the dramatic drop in vulnerability (at 8 vs 16 weeks) and even when the initial exposure led to threshold shifts, which were completely reversible in the short term (Fig. 1). Similar AHL/NIHL interactions are seen in the data at $16 \mathrm{kHz}$, except for the group exposed at 4 weeks wherein the threshold shifts may saturate, thus leading to an underestimate of the "additional" threshold shifts as these animals age.

\section{AHL/NIHL interactions produce primary neural degeneration}

When cochleae from young- or old-exposed animals were harvested 2 weeks after exposure and examined with age-matched, unexposed counterparts, the organ of Corti was intact; there was virtually no hair cell loss outside of the extreme high-frequency end of the cochlear spiral. Other cochlear structures, including the stria vascularis, spiral limbus, tectorial membrane, etc., did not appear pathologic in any systematic way (Figs. 5A, 6A-C). Such results are consistent with previous work (Liberman and Beil, 1979) demonstrating that noise-induced permanent threshold shifts on the order of $40 \mathrm{~dB}$ do not require hair cell loss but can occur with stereocilia damage alone (which was not evaluated in the present study).

Many of the noise-exposed ears showed cell loss among a small spatially distinct class of fibrocytes (type IV) within the spiral liga- 

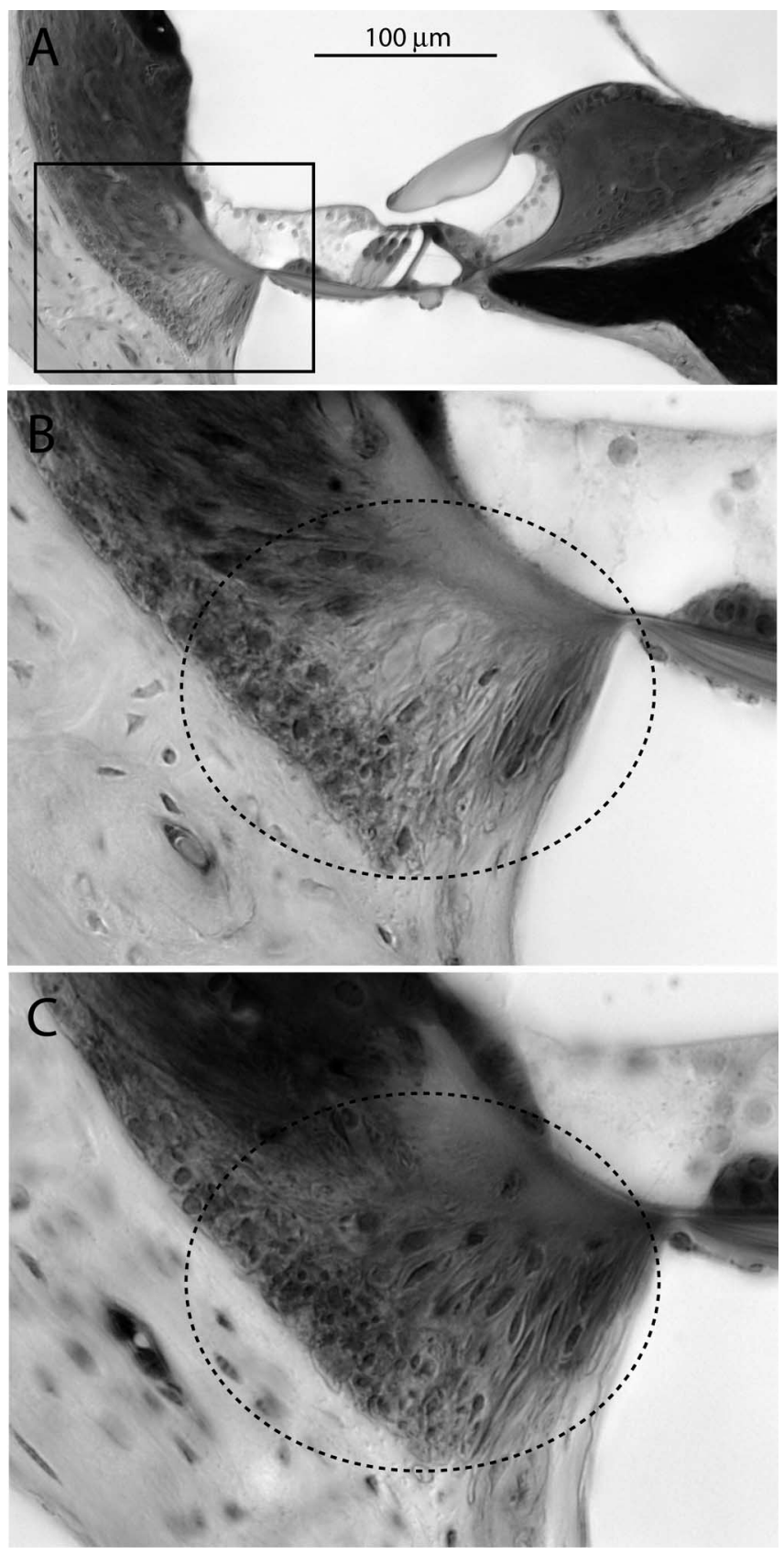

Figure 5. When examined 2 weeks after exposure, the only histopathology is loss of type IV fibrocytes: compare circled regions of $\boldsymbol{B}$ and $\boldsymbol{C}$. $\boldsymbol{A}$ and $\boldsymbol{B}$ show the upper basal turn of an ear exposed at 6 weeks and tissues processed at 8 weeks. The region of the high-power view in $B$ is indicated by the box in $\boldsymbol{A}$. C shows the normal appearance of the type IV fibrocytes at the same cochlear region.

ment (Fig. 5, compare $B, C$ ). The presence or absence of type IV cells was not well correlated with the degree of threshold shift: as shown in Figure 8, estimated type IV loss was greater in ears exposed at 2 years than in those exposed at 6 weeks, although the former had no permanent NIHL, whereas the latter had a $40 \mathrm{~dB}$ shift. Previous studies have noted the vulnerability of the type IV cells and the lack of correlation with threshold shifts (Wang et al., 2002).

The most striking histopathologic change was a widespread loss of spiral ganglion cells, the cell bodies of cochlear nerve afferents, most of which make synaptic contact with inner hair cells. As illustrated by the micrographs in Figure 6, such neuronal loss was seen only in aged ears that were noise-exposed earlier in life; it was not seen in unexposed groups, regardless of age, nor in noise-exposed groups evaluated at short postexposure times. Because the neuronal loss was not associated with hair cell loss, it is considered a "primary" neural degeneration rather than occurring secondary to the hair cell degeneration. Even at the limits of resolution of the light microscope, the hair cells and supporting cells of the organ of Corti looked completely normal in most cochlear regions of all exposure groups: representative highpower differential interference contrast (DIC) images are shown in Figure 7. Based on previous studies at both the light and electron microscopic levels (Liberman and Dodds, 1984), it is likely that initial noise-induced threshold shift in our mice was attributable to stereocilia damage, especially on outer hair cells, which is not well resolved in the type of histological material used in this study.

These qualitative observations were quantified and systematically evaluated in an analysis by an observer blind to the age and exposure history of the tissue. Results of this analysis are shown in Figure 8, in which means and SEs are shown for fractional survival of inner and outer hair cells, spiral ganglion cells, and type IV fibrocytes in ears from representative groups in the present study. None of the groups showed significant loss of inner hair cells, and variable amounts of outer hair cell loss were seen but only in the more apical regions (primarily 6 and $14 \mathrm{kHz}$ ) and only in the older animals (regardless of exposure history). Apical outer hair cell loss has been reported previously in aging $\mathrm{CBA} / \mathrm{CaJ}$ (Henry and Chole, 1980; Spongr et al., 1997) and CBA/J (Ohlemiller and Gagnon, 2004). Type IV fibrocyte loss was seen primarily in the high-frequency region $(30 \mathrm{kHz})$ and only in the noise-exposed ears, with severity of loss greatest in ears surviving for longer postexposure times. Widespread loss of spiral ganglion cells was seen only in noise-exposed animals held for long postexposure times. In unexposed animals, there was modest loss $(<20 \%$ on average) but only in the apex and only in old animals, consistent with previous studies of aging mice and rats (Keithley and Feldman, 1979, 1982; Dazert et al., 1996). In exposed animals, ganglion cell loss was not seen in the short-surviving ears, whether the age at exposure was 6 weeks ("Expose Young Test Young") or 124 weeks ("Expose Old Test Old"). In the long-surviving ears, the neuronal loss could be seen throughout the cochlea and was significant regardless of whether the ears were exposed at 6 weeks ("Expose Young Test Old") or at 16-32 weeks (data not shown).

\section{Discussion}

\section{Critical period for noise vulnerability}

Numerous studies of cochlear function have suggested a period of heightened sensitivity to insult from noise or ototoxic drugs during (Bock and Saunders, 1977; Bock and Seifter, 1978; Lenoir et al., 1979; Henley and Rybak, 1993) and beyond (Henry, 1982, 1983; Pujol, 1992) periods of obvious structural and functional maturation of the cochlea. In mouse, age-related shifts in NIHL vulnerability are well documented. In commonly used variants of CBA (CBA/J, CBA/Ca, and CBA/CaJ), noise vulnerability decreases with age (Henry, 1982, 1983; Li and Borg, 1993; Ohlemiller et al., 2000). Henry (1982) exposed CBA mice at $60,90,120$, and $360 \mathrm{~d}$ and found a reduction in vulnerability as exposure age increased from 60 to $120 \mathrm{~d}$ (nominally 8 and 17 weeks). Present results are in good agreement with this observation.

Middle ear motion decreases with age (Doan et al., 1996; Rosowski et al., 2003), and less efficient transfer through the middle ear could decrease NIHL vulnerability. Direct measurements of sound transmission through the aging middle ear in $\mathrm{CBA} / \mathrm{CaJ}$ (Rosowski et al., 2003) show that transmission losses may account for part of the sensitivity reduction $(<6 \mathrm{~dB})$ at frequencies above 16 
$\mathrm{kHz}$ in old, unexposed ears. However, in the noise-exposure band used here $(8-16 \mathrm{kHz})$, middle ear transmission changes by $<2 \mathrm{~dB}$ between 8 and 96 weeks. Middle ear changes between 8 and 16 weeks must be significantly smaller and cannot underlie the dramatic change in noise vulnerability. Even a $2 \mathrm{~dB}$ transmission decrease could cause a $12 \mathrm{~dB}$ decrease in NIHL (Yoshida et al., 2000), i.e., much smaller than the $40 \mathrm{~dB}$ differences observed between 8 and 16 week animals (Fig. 1). Thus, the age-related shift in vulnerability must arise in the inner ear.

The inner ear is mature morphologically, and electrophysiologic properties of hair cells and gross cochlear response thresholds have stabilized by 4 weeks, the youngest animals studied here (Mikaelian et al., 1965; Lim and Anniko, 1985; Marcotti et al., 2003; Hafidi et al., 2005). Thresholds in the 8-16 $\mathrm{kHz}$ range are almost identical in 4 - versus 16-week-old animals (Fig. 2); thus, the vulnerability shift has no obvious correlate in cochlear sensitivity. Indeed, apart from the dramatic shift in noise vulnerability, there is no reported change in cochlear structure or function over the critical time period from 8 to 16 weeks. It may be significant that mice reach sexual maturity $\sim 6-8$ weeks (first in females and then males); thus, endocrine changes may produce previously undetected changes in cochlear function that influence noise vulnerability. In the present series, no difference in preexposure thresholds was seen for young males versus females. Two studies, those of Guimaraes et al. (2004) and Henry (2004), report that, among older CBA mice, males show higher thresholds (i.e., more AHL) than females. Although no gender difference in noise vulnerability was observed here for short postexposure times, evaluation of possible gender influences on the progressive neuropathy of long-surviving animals is underway.

\section{Interactions between noise and age in animal models}

Studies of acoustic trauma (Miller et al., 1963) suggest the following: (1) noiseinduced threshold shift increases only as long as the noise exposure continues, (2) threshold recovery begins soon after exposure termination, and (3) noise-induced threshold shift asymptotes to permanent and stable levels within 2-4 weeks after exposure. Findings from the present study challenge the universality of these notions. Specifically, we show that noise exposure can lead to threshold shifts that progress for years after the exposure and are associated with primary degeneration of the cochlear nerve. This neural etiology contrasts with the noise-induced hair cell (or stereocilia) damage that underlies the bar in $\boldsymbol{A}$ applies to $\boldsymbol{A}-\boldsymbol{D}$.
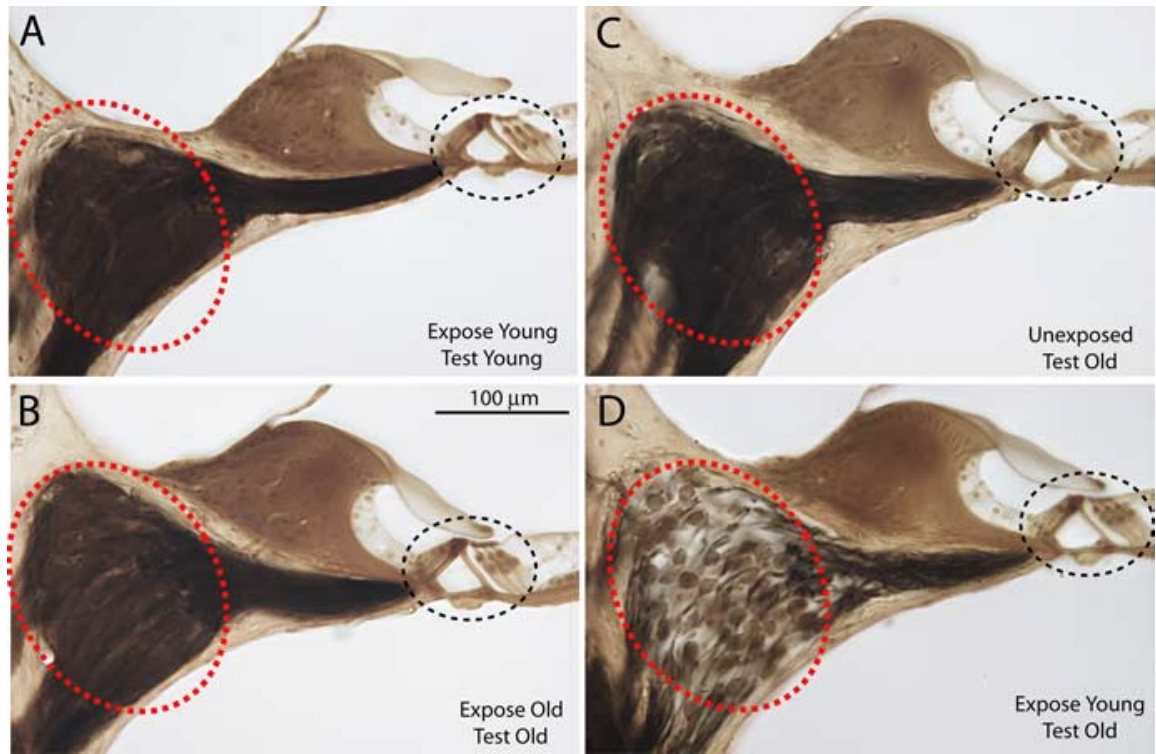

Figure 6. Primary neuronal degeneration was seen in mice that were exposed and allowed to survive for many months. The degeneration, seen as decreased density of spiral ganglion cells (heavy black circles), although inner and outer hair cells (light black circles) are still present, is visible in cases exposed at 6 weeks and aged to 96 weeks (D) but not in cases exposed at 96 weeks and evaluated at 98 weeks $(\boldsymbol{B})$ or in unexposed animals tested at 96 weeks $(\boldsymbol{C})$ or in cases exposed at 6 weeks and tested at 8 weeks (A). All images are from the upper basal turn. Scale bar in $\boldsymbol{B}$ applies to $\boldsymbol{A}-\boldsymbol{D}$.
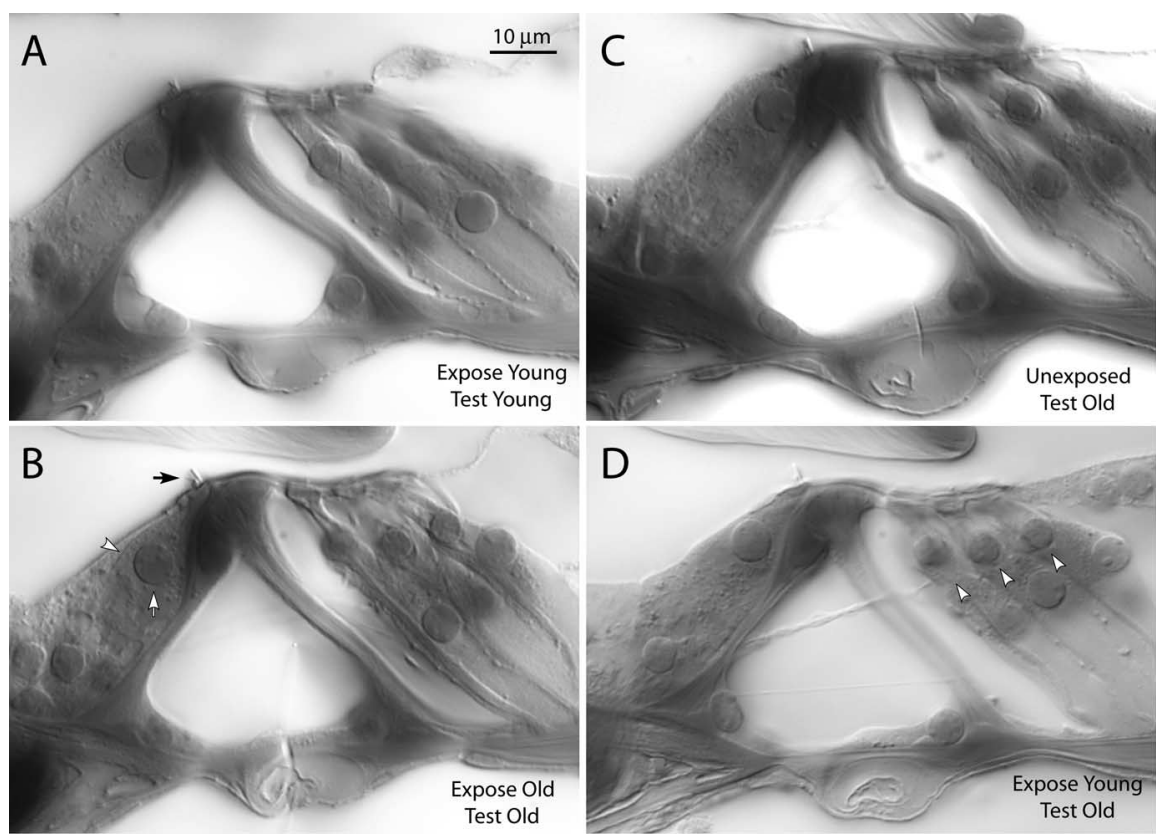

Figure 7. The sensory epithelium appears normal in animals from all exposure groups, even with high-power DIC optics. Images are from the same four cases shown in Figure 6. Each image is focused on an inner hair cell nucleus (e.g., white arrow in $\boldsymbol{B}$ ). Stereocilia on inner hair cells are also in focus (e.g., black arrow in $\boldsymbol{B}$ ), and, in some cases, the basolateral membrane of the inner hair cell is visible (e.g., white arrowhead in $\boldsymbol{B}$ ). Three rows of outer hair cells are seen in all images (e.g., white arrows in $\boldsymbol{D}$ ); however, not all rows are in focus. Outer hair cell stereocilia in mouse are generally too small to be visible in this material. The scale

initially measured permanent threshold shifts (Liberman and Dodds, 1984) and contrasts with the aging process in unexposed mice, as shown here and by other studies reporting that cochlear neuronal loss is minimal in unexposed mice, even beyond 2 years of age (Lambert and Schwartz, 1982; Willott et al., 1988; Ohlemiller and Gagnon, 2004).

The present study does not address mechanisms underlying 


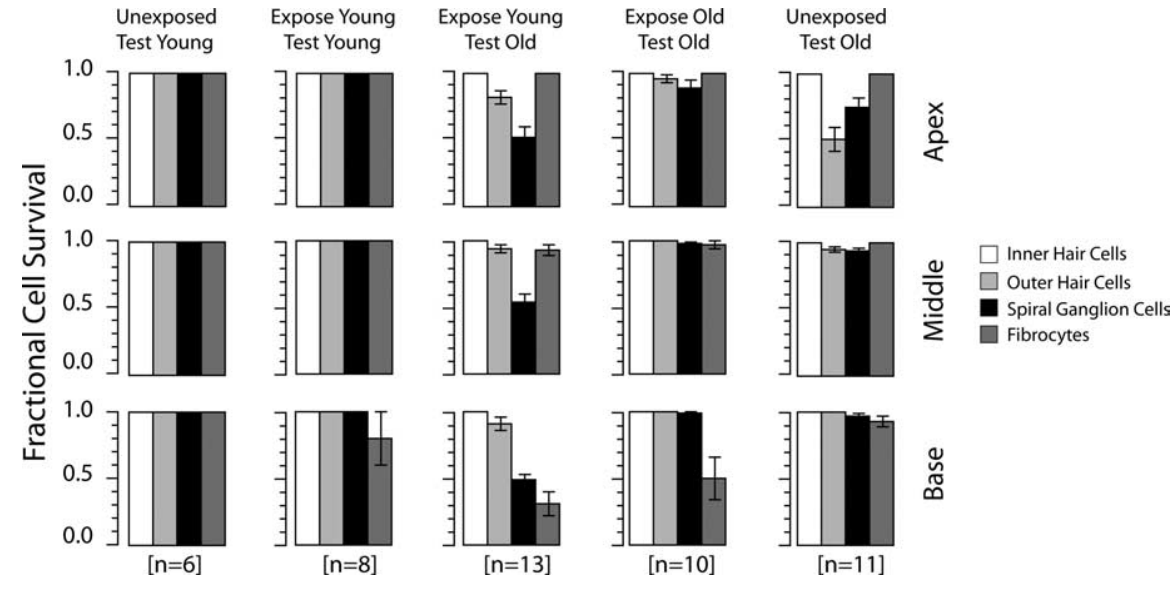

Figure 8. Semiquantitative analysis of cochlear histopathology in groups of exposed and unexposed animals performed by an observer blind to the exposure history and threshold measures. Analysis included estimates of inner and outer hair cell loss, spiral ganglion cell loss, and loss of type IV fibrocytes. Each histogram shows means and SEs or the estimates of fractional cell survival. Estimates were made in three cochlear regions, as indicated, corresponding to the three regions seen in a midcochlear section. The numbers of animals in each group are given under each column letter. "Unexposed Test Young" animals were tested at 7.5 weeks; "Expose Young Test Young" were exposed at 5.5 weeks and tested at 7.5 weeks; "Expose Young Test Old" animals were exposed at 5 weeks and tested at 100 weeks; "Expose 0ld Test 0ld" animals were exposed at 124 weeks and tested at 126 weeks; and "Unexposed Test Old" animals were tested at 105 weeks.

the progressive neuropathy; however, it may be important in this regard that the acute response to noise often includes not only temporary threshold shifts but evidence of glutamate excitotoxicity [swelling of afferent nerve terminals under inner hair cells (Pujol et al., 1993)]. Indeed, exposures identical to those used here, delivered at 10 weeks to mice of the same strain, caused temporary threshold shifts that extended across the entire range of frequencies monitored $(5.6-45.2 \mathrm{kHz})$ and obvious swelling of dendrites and cell bodies of cochlear neurons along a broad extent of the cochlear epithelium when evaluated $24 \mathrm{~h}$ after exposure (Wang et al., 2002). Although the swelling subsides by 1 week after exposure and synaptic function can return to normal, as evidenced by recovery of ABR thresholds, present results suggest that long-term changes are set in motion that can lead to degeneration on a timescale of months to years.

Progressive threshold deterioration was worst for animals exposed during the "critical period," although some threshold deterioration (and significant associated neuropathy) beyond that expected by aging alone also was seen in animals exposed at older ages and held many months after exposure. This is remarkable because the older animals showed virtually no threshold shift 2 weeks after exposure. Thus, long-term effects of noise exposure can be documented even after an exposure that initially appears to be fully reversible.

Few other studies have followed animals for long postexposure times. However, the two most relevant previous studies, including one in mouse (Li and Borg, 1993) and one in gerbil (Mills et al., 1997), found no evidence for progressive threshold shifts in noise-exposed animals above and beyond those seen in age-matched controls. Histopathology was not evaluated in either case. In the earlier mouse study, CBA/CaJ at 1-12 months were exposed to a $5 \mathrm{~min}, 120 \mathrm{~dB}$ SPL, $2-7 \mathrm{kHz}$ noise band that caused an initial permanent shift of $\sim 20 \mathrm{~dB}$ and were held to 23-27 months of age for final ABR testing. In the gerbil study, animals were exposed at 18 months to a $3.5 \mathrm{kHz}$ pure tone for $1 \mathrm{~h}$ at $113 \mathrm{~dB}$ SPL that caused an initial permanent shift of $\sim 20 \mathrm{~dB}$ and were allowed to survive for an additional 18 months before final ABR testing. Apparent discrepancies with current findings may arise from several sources. In the gerbil study, animals were exposed at middle age: in the present study, middle-aged animals held to old age also showed little additional threshold shift. In the mouse study, the short-duration, high-level, lowfrequency exposure stimulus may initiate different pathologic processes than those produced here. For example, the loss of spiral ligament fibrocytes seen by 2 weeks after exposure in our animals may not occur after a 5 min exposure such as that used by Li and Borg (1993). Given that spiral ligament fibrocytes may be involved in cytokine signaling pathways and thereby in the stress response of the ear (Adams, 2002), the loss of fibrocytes in our animals may be key in the initiation of long-term neural degeneration.

The magnitude of the ABR shifts in the long-surviving noise-exposed animals is too large to be accounted for by the spiral ganglion cell loss alone. Good thresholds for tones can be maintained after primary neural lesion (Schuknecht, 1993; Parkinson et al., 2001) or neural loss to selective inner hair cell degeneration (Schrott et al., 1989; Wake et al., 1993; Liberman et al., 1997; Hamernik et al., 1998). In addition to the ganglion cells that are frankly missing in these ears, there may be large numbers of unresponsive neurons, perhaps because of degeneration of their peripheral terminals on inner hair cells, as suggested from ultrastructural studies of human temporal bones (Nadol, 1988).

\section{Implications for presbycusis and hearing loss allocation in humans}

Aging humans lose threshold sensitivity, especially at high frequencies, and show increasing difficulties discriminating speech in noisy environments. This syndrome of age-related decrements in auditory performance is called presbycusis. For a significant subset of aging individuals, performance on these two functional metrics diverges, with speech intelligibility losses outweighing those expected from threshold sensitivity declines (Pauler et al., 1986). Such performance deficits, together with histologic evidence of primary neural degeneration in some aging ears, have suggested a "neural presbycusis" (Schuknecht, 1993; Schuknecht and Gacek, 1993). Thus, primary neural degeneration of the type seen in the present study could have important consequences in the human, even if the changes in pure tone thresholds are not large.

Current clinical practice and medico-legal procedures often require allocation of noise-induced versus age-related components of hearing losses in aging ears (Dobie, 1992). Methods commonly used to aid such allocations treat these components as though they add simply (with some compression for large shifts) in their contribution to the aggregate hearing loss recorded in a given ear (International Organization for Standardization, 1990, 1999) (see also American College of Occupational Medicine, 1989). This notion has found support in a recent longitudinal study (Lee et al., 2005) reporting that threshold shifts over 3-11.5 year timespans were not significantly different for individuals with and without reported noise-exposure histories. However, two recent studies have yielded data that contradict this view (Gates et al., 2000; Rosenhall, 2003). In the Gates et al. study, ears 
with large 3-6 kHz reductions in threshold sensitivity [taken as evidence of previous noise damage (Cooper and Owen, 1976; Kryter, 1985)] demonstrated age-noise interactions resulting in additional hearing loss progression primarily in frequency regions below the original noise-induced threshold shift. Similar changes with age were not seen in ears without these noise notches. This finding was subsequently confirmed by Rosenhall (2003) in analysis of the annual decline of pure tone thresholds in aging men with versus without reported histories of occupational noise exposure. Such observations and those tested more directly here suggest that ears with noise-exposure histories age differently from those without and that the mouse may be a useful animal model in which to systematically study these important issues.

\section{References}

Adams JC (2002) Clinical implications of inflammatory cytokines in the cochlea: a technical note. Otol Neurotol 23:316-322.

American College of Occupational Medicine (1989) Occupational noiseinduced hearing loss. ACOM Noise and Hearing Conservation Committee. J Occup Med 31:996-1001.

Bock GR, Saunders JC (1977) A critical period for acoustic trauma in the hamster and its relation to cochlear development. Science 197:396-398.

Bock GR, Seifter EJ (1978) Developmental changes of susceptibility to auditory fatigue in young hamsters. Audiology 17:193-203.

Candreia C, Martin GK, Stagner BB, Lonsbury-Martin BL (2004) Distortion product otoacoustic emissions show exceptional resistance to noise exposure in MOLF/Ei mice. Hear Res 194:109-117.

Cooper JC, Owen JH (1976) Audiologic profile of noise-induced hearing loss. Arch Otolaryngol 102:148-150.

Dallos P (1992) The active cochlea. J Neurosci 12:4575-4585.

Davis RR, Kozel P, Erway LC (2003) Genetic influences in individual susceptibility to noise: a review. Noise Health 5:19-28.

Dazert S, Feldman ML, Keithley EM (1996) Cochlear spiral ganglion cell degeneration in wild-caught mice as a function of age. Hear Res 100:101-106.

DeStefano AL, Gates GA, Heard-Costa N, Myers RH, Baldwin CT (2003) Genomewide linkage analysis to presbycusis in the Framingham Heart Study. Arch Otolaryngol Head Neck Surg 129:285-289.

Doan DE, Erulkar JS, Saunders JC (1996) Functional changes in the aging mouse middle ear. Hear Res 97:174-177.

Dobie RA (1992) The relative contributions of occupational noise and aging in individual cases of hearing loss. Ear Hear 13:19-27.

Erway LE, Willott JF, Archer JR, Harrison DE (1993) Genetics of age-related hearing loss in mice. I. Inbred and F1 hybrid strains. Hear Res 65:125-132.

Folmer RL, Griest SE, Martin WH (2002) Hearing conservation education programs for children: a review. J Sch Health 72:51-57.

Gates G, Mills J (2005) Presbycusis. Lancet 366:1111-1120.

Gates GA, Couropmitree NN, Myers RH (1999) Genetic associations in agerelated hearing thresholds. Arch Otolaryngol Head Neck Surg 125:654-659.

Gates GA, Schmid P, Kujawa SG, Nam B, D’Agostino R (2000) Longitudinal threshold changes in older men with audiometric notches. Hear Res 141:220-228.

Guimaraes P, Zhu X, Cannon T, Kim S, Frisina RD (2004) Sex differences in distortion product otoacoustic emissions as a function of age in CBA mice. Hear Res 192:83-89.

Hafidi A, Beurg M, Dulon D (2005) Localization and developmental expression of BK channels in mammalian cochlear hair cells. Neuroscience 130:475-484.

Hamernik RP, Patterson JH, Turrentine GA, Ahroon WA (1989) The quantitative relation between sensory cell loss and hearing thresholds. Hear Res 38:199-211

Hamernik RP, Ahroon WA, Jock BM, Bennett JA (1998) Noise-induced threshold shift dynamics measured with distortion-product otoacoustic emissions and auditory evoked potentials in chinchillas with inner hair cell deficient cochleas. Hear Res 118:73-82.

Henderson D, Subramaniam M, Boettcher FA (1993) Individual susceptibility to noise-induced hearing loss: an old topic revisited. Ear Hear 14:152-168

Henley CM, Rybak LP (1993) Developmental ototoxicity. Otolaryngol Clin North Am 26:857-871.
Henry KR (1982) Age-related changes in sensitivity of the postpubertal ear to acoustic trauma. Hear Res 8:285-294.

Henry KR (1983) Lifelong susceptibility to acoustic trauma: changing patterns of cochlear damage over the life span of the mouse. Audiology 22:372-383.

Henry KR (2004) Males lose hearing earlier in mouse models of late-onset age-related hearing loss; females lose hearing earlier in mouse models of early-onset hearing loss. Hear Res 190:141-148.

Henry KR, Chole RA (1980) Genotypic differences in behavioral, physiological and anatomical expressions of age-related hearing loss in the laboratory mouse. Audiology 19:369-383.

Hequembourg S, Liberman MC (2001) Spiral ligament pathology: a major aspect of age-related cochlear degeneration in C57BL/6 mice. J Assoc Res Otolaryngol 2:118-129.

Hunter KP, Willott JF (1987) Aging and the auditory brainstem response in mice with severe or minimal presbycusis. Hear Res 30:207-218.

International Organization for Standardization (1990, 1999) Acousticsdetermination of occupational noise exposure and estimation of noiseinduced hearing impairment. Geneva: International Organization for Standardization.

Jimenez AM, Stagner BB, Martin GK, Lonsbury-martin BL (1999) Agerelated loss of distortion product otoacoustic emissions in four mouse strains. Hear Res 138:91-105.

Johnson KR, Erway LC, Cook SA, Willott JF, Zheng QY (1997) A major gene affecting age-related hearing loss in C57BL/6J mice. Hear Res 114:83-92.

Karlsson K, Harris JR, Svartengren M (1997) Description and primary results from an audiometric study of male twins. Ear Hear 18:114-120.

Keithley EM, Feldman ML (1979) Spiral ganglion cell counts in an agegraded series of rat cochleas. J Comp Neurol 188:429-442.

Keithley EM, Feldman ML (1982) Hair cell counts in an age-graded series of rat cochleas. Hear Res 8:249-262.

Kryter KD (1985) The effects of noise on man, Ed 2. New York: Academic.

Kujawa SG, Liberman MC (1999) Long-term sound conditioning enhances cochlear sensitivity. J Neurophysiol 82:863-873.

Lambert PR, Schwartz IR (1982) A longitudinal study of changes in the cochlear nucleus in the CBA mouse. Otolaryngol Head Neck Surg 90:787-794.

Lee FS, Matthews LJ, Dubno JR, Mills JH (2005) Longitudinal study of puretone thresholds in older persons. Ear Hear 26:1-11.

Lenoir M, Bock G, Pujol R (1979) Supra-normal susceptibility to acoustic trauma of the rat pup cochlea. J Physiol (Paris) 75:521-524.

Li HS (1992) Genetic influences on susceptibility of the auditory system to aging and environmental factors. Scand Audiol Suppl 36:1-39.

Li HS, Borg E (1993) Auditory degeneration after acoustic trauma in two genotypes of mice. Hear Res 68:19-27.

Liberman MC, Beil DG (1979) Hair cell condition and auditory nerve response in normal and noise-damaged cochleas. Acta Otolaryngol 88:161-176.

Liberman MC, Dodds LW (1984) Single-neuron labeling and chronic cochlear pathology. III. Stereocilia damage and alterations of threshold tuning curves. Hear Res 16:55-74.

Liberman MC, Chesney CP, Kujawa SG (1997) Effects of selective inner hair cell loss on DPOAE and CAP in carboplatin-treated chinchillas. Aud Neurosci 3:255-268.

Lim DJ, Anniko M (1985) Developmental morphology of the mouse inner ear. A scanning electron microscopic observation. Acta Otolaryngol Suppl 422:1-69.

Lutman ME, Spencer HS (1990) Occupational noise and demographic factors in hearing. Acta Otolaryngol Suppl 476:74-88.

Marcotti W, Johnson SL, Holley MC, Kros CJ (2003) Developmental changes in the expression of potassium currents of embryonic, neonatal and mature mouse inner hair cells. J Physiol (Lond) 548:383-400.

Mikaelian D, Alford BR, Ruben RJ (1965) Cochlear potentials and 8 nerve action potentials in normal and genetically deaf mice. Ann Otol Rhinol Laryngol 74:146-157.

Miller JD, Watson CS, Covell WP (1963) Deafening effects of noise on the cat. Acta OtoLaryngol Suppl 176:1-91.

Mills JH, Boettcher FA, Dubno JR (1997) Interaction of noise-induced permanent threshold shift and age-related threshold shift. J Acoust Soc Am 101:1681-1686.

Nadol Jr JB (1988) Application of electron microscopy to human otopathol- 
ogy. Ultrastructural findings in neural presbycusis, Meniere's disease and Usher's syndrome. Acta Otolaryngol 105:411-419.

National Institutes of Health (2000) Healthy people 2010: focus area 28, Vision and hearing. Rockville, MD: Office of Disease Prevention and Health Promotion, U.S. Department of Health and Human Services.

Ohlemiller KK, Gagnon PM (2004) Apical-to-basal gradients in age-related cochlear degeneration and their relationship to "primary" loss of cochlear neurons. J Comp Neurol 479:103-116.

Ohlemiller KK, Wright JS, Heidbreder AF (2000) Vulnerability to noiseinduced hearing loss in "middle-aged" and young adult mice: a doseresponse approach in CBA, C57BL, and BALB inbred strains. Hear Res 149:239-247.

Parkinson NJ, Olsson CL, Hallows JL, McKee-Johnson J, Keogh BP, NobenTrauth K, Kujawa SG, Tempel BL (2001) Mutant beta-spectrin 4 causes auditory and motor neuropathies in quivering mice. Nat Genet 29:61-65.

Pauler M, Schuknecht HF, Thornton AR (1986) Correlative studies of cochlear neuronal loss with speech discrimination and pure-tone thresholds. Arch Otorhinolaryngol 243:200-206.

Pujol R (1992) Sensitive developmental period and acoustic trauma: facts and hypotheses. In: Noise-induced hearing loss (Dancer AL, Henderson D, Salvi RJ, Hamernik RP, eds), pp 196-203. St. Louis: Mosby.

Pujol R, Puel JL, Gervais d'Aldin C, Eybalin M (1993) Pathophysiology of the glutamatergic synapses in the cochlea. Acta Otolaryngol 113:330-334.

Rosenhall U (2003) The influence of ageing on noise-induced hearing loss. Noise Health 5:47-53.

Rosowski JJ, Brinsko KM, Tempel BI, Kujawa SG (2003) The aging of the middle ear in 129S6/SvEvTac and CBA/CaJ mice: measurements of umbo velocity, hearing function, and the incidence of pathology. J Assoc Res Otolaryngol 4:371-383.
Saunders JC, Cohen YE, Szymko YM (1991) The structural and functional consequences of acoustic injury in the cochlea and peripheral auditory system: a five year update. J Acoust Soc Am 90:136-146.

Schrott A, Stephan K, Spoendlin H (1989) Hearing with selective inner hair cell loss. Hear Res 40:213-219.

Schuknecht HF (1993) Pathology of the ear, Ed 2. Philadelphia: Lea and Febiger.

Schuknecht HF, Gacek MR (1993) Cochlear pathology in presbycusis. Ann Otol Rhinol Laryngol 102:1-16.

Spongr VP, Flood DG, Frisina RD, Salvi RJ (1997) Quantitative measures of hair cell loss in CBA and C57BL/6 mice throughout their life spans. J Acoust Soc Am 101:3546-3553.

Wake M, Takeno S, Ibrahim D, Harrison R, Mount R (1993) Carboplatin ototoxicity: an animal model. J Laryngol Otol 107:585-589.

Wallhagen MI, Strawbridge WJ, Cohen RD, Kaplan GA (1997) An increasing prevalence of hearing impairment and associated risk factors over three decades of the Alameda County Study. Am J Public Health $87: 440-442$.

Wang Y, Hirose K, Liberman MC (2002) Dynamics of noise-induced cellular injury and repair in the mouse cochlea. J Assoc Res Otolaryngol 3:248-268.

Willott JF, Parham K, Hunter KP (1988) Response properties of inferior colliculus neurons in young and very old CBA/J mice. Hear Res 37:1-14.

Yoshida N, Hequembourg SJ, Atencio CA, Rosowski JJ, Liberman MC (2000) Acoustic injury in mice: $129 / \mathrm{SvEv}$ is exceptionally resistant to noise-induced hearing loss. Hear Res 141:97-106.

Zheng QY, Johnson KR, Erway LC (1999) Assessment of hearing in 80 inbred strains of mice by ABR threshold analyses. Hear Res 130: 94-107. 